(2) Open Access Full Text Article

REVIEW

\title{
Macular edema in the era of spectral-domain optical coherence tomography
}

This article was published in the following Dove Press journal:

Clinical Ophthalmology

17 October 2013

Number of times this article has been viewed

\author{
Allan Hunter' \\ Eric K Chin' \\ David G Telander ${ }^{2}$ \\ 'Department of Ophthalmology, \\ University of California, Davis, \\ ${ }^{2}$ Department of Ophthalmology, \\ Retinal Consultants, Sacramento, \\ CA, USA
}

Correspondence: Allan Hunter University of California, Davis, 4860 Y Street, Suite 2400, Sacramento, CA 95817, USA

$\mathrm{Tel}+\mathrm{I} 9167346602$

Fax + I 9167346074

Email hunter.allan.a@gmail.com

\begin{abstract}
The development of spectral-domain optical coherence tomography (OCT) allows for the highest commercially available resolution of in vivo retinal anatomic details to date. The ability to see the macula with ever increasing detail is dramatically improving our understanding of the pathogenesis of retinal disease. However, the only prospective study that partially evaluated spectral-domain OCT versus time-domain OCT failed to show any clinical benefit of increased OCT resolution. Clinical outcomes, eg, best-corrected visual acuity, central macular thickness and number of injections, with "newer" OCT technologies remain an unproven advantage.

Keywords: retina, macular edema, optical coherence tomography, fluorescein angiography, indocyanine green angiography
\end{abstract}

\section{Introduction}

Understanding of retinal disease is limited by the lack of retinal histology with detailed pre-mortem clinical corroboration of disease state and severity. Histopathologic studies created the foundation of our understanding of retinal pathology, but could not provide in vivo information to clarify cellular responses to treatment. To augment our understanding, ophthalmologists have used slit-lamp biomicroscopy, fundus photography, autofluorescence, fluorescein angiography, and indocyanine green angiography. Although detailed, this has only given ophthalmologists a nonhistologic description of retinal pathology. However, since the first description of optical coherence tomography (OCT) by Huang et al in 1991, the in vivo anatomic illustration of pathology has been immeasurably important to our basic understanding of retinal disease and response to treatment, especially macular diseases like cystoid macular edema. ${ }^{1}$

Historically, the first widely used systems were time-domain OCT technologies. With the release of the newer spectral-domain images, spectral-domain OCT has the advantage of improved image quality, resolution, and faster acquisition times (Table 1). ${ }^{2}$ OCT imaging allows specialists to assess accurately the location and extent of retinal changes and objectively determine axial (depth) and transverse (surface area) measurements. Spectral-domain OCT uses a high-speed spectrometer to measure light echoes from all time delays simultaneously, which has many advantages over its time-domain OCT predecessors. It does not require a reference mirror to be mechanically scanned, which allows for improved sensitivity, faster sampling speed, and optimal signal-tonoise ratio. Commercially available devices achieve image acquisition speeds of greater than 40,000 A-scans per second, with an axial resolution of approximately 4-7 microns. With greater resolution power, smaller morphologic changes are identifiable. ${ }^{3}$ Static 
Table I Image characteristics of optical coherence tomography

\begin{tabular}{llll}
\hline $\begin{array}{l}\text { Type } \\
\text { of OCT }\end{array}$ & $\begin{array}{l}\text { Axial } \\
\text { resolution }\end{array}$ & $\begin{array}{l}\text { A-scans per } \\
\text { second }\end{array}$ & Topography \\
\hline TD & $10 \mu \mathrm{m}$ & 400 & Six radial scans \\
SD & $>3 \mu \mathrm{m}$ & $<40,000$ & Three-dimensional \\
& & & reconstructions \\
\hline
\end{tabular}

Abbreviations: TD, time-domain; SD, spectral-domain; OCT, optical coherence tomography.

retinal anatomic images can now help to describe dynamic pathologic processes through serial spectral-domain OCT image acquisitions.

The precise role of spectral-domain OCT in the diagnosis of macular edema is evolving, and it remains unclear if this technique complements or replaces the imaging modalities previously available to ophthalmologists. For example, the gold standard for evaluating diabetic macular edema has been contact fundus biomicroscopy. Brown et al found excellent agreement between foveal thickening on OCT and contact lens assessment when foveal thickness was either normal (less than 200 microns) or moderate to severely increased (greater than 300 microns). ${ }^{4}$ However, a substantially decreased level of agreement was found with mild macular edema, where foveal thickness ranges between 201 and 300 microns. The authors concluded that OCT is more sensitive for detecting the presence of diabetic macular edema in eyes with "mild" macular edema. Moreover, several studies have shown that spectral-domain OCT has greater sensitivity for detecting cystoid macular edema in its early stages prior to visualization by detailed ophthalmoscopy or fluorescein angiography. ${ }^{5-8}$ In addition, spectral-domain OCT can help clinicians and researchers to determine the treatment effect in a quantitative manner.

\section{Spectral-domain OCT in diagnosis of macular edema}

The anatomic findings of cystoid macular edema demonstrated by spectral-domain OCT are described by their degree of reflectivity. The causes of hyper-reflective lesions vary according to the pathology. Cotton wool spots and extracellular exudates are hyper-reflective OCT findings in diabetic retinopathy and retinal vein occlusion. Epiretinal membranes are also hyper-reflective and commonly cause cystoid macular edema and photoreceptor damage. In central serous chorioretinopathy and parafoveal telangiectasia, retinal pigment epithelial hyperplasia can be visualized as hyper-reflective signals. Hyporeflective areas in an OCT retinal image usually represent areas of extracellular fluid found in cystoid macular edema from many causes, including diabetic maculopathy, retinal vein occlusion, or inflammation. A shadow effect can be seen with hemorrhage, exudate, dense epiretinal membrane, and "flow void" beneath the retinal vessels.

Perhaps the most significant function of spectral-domain OCT is its ability to clarify the status of the outer retina. By visualizing the outer plexiform layer, outer nuclear layer, external limiting membrane, inner/outer segment (IS/OS) of photoreceptors, outer segments of photoreceptors (also known as Verhoeff's membrane), OCT allows an anatomic correlation with vision (Figure 1). The status of the photoreceptor IS/OS and external limiting membrane is helpful in predicting the final visual outcome in macular edema associated with branch retinal vein occlusion. ${ }^{9}$ In fact, changes in the outer retina seen on spectral-domain OCT have been found to help explain visual outcomes after repair of retinal detachment and many cases of unexplained visual loss..$^{10,11}$

Numerous studies have correlated best-corrected visual acuity (BCVA) with retinal thickness or loss of anatomic distinction of the outer retina, starting with Otani et al. ${ }^{12}$ There is a modest correlation between central macular thickness and visual acuity measured on OCT. ${ }^{13}$ The Diabetic Retinopathy Clinical Research Network (DRCR.net) produced the largest study analyzing the correlation between central retinal thickness and visual acuity. Interestingly, that study found a paradoxical improvement in vision with increased thickening

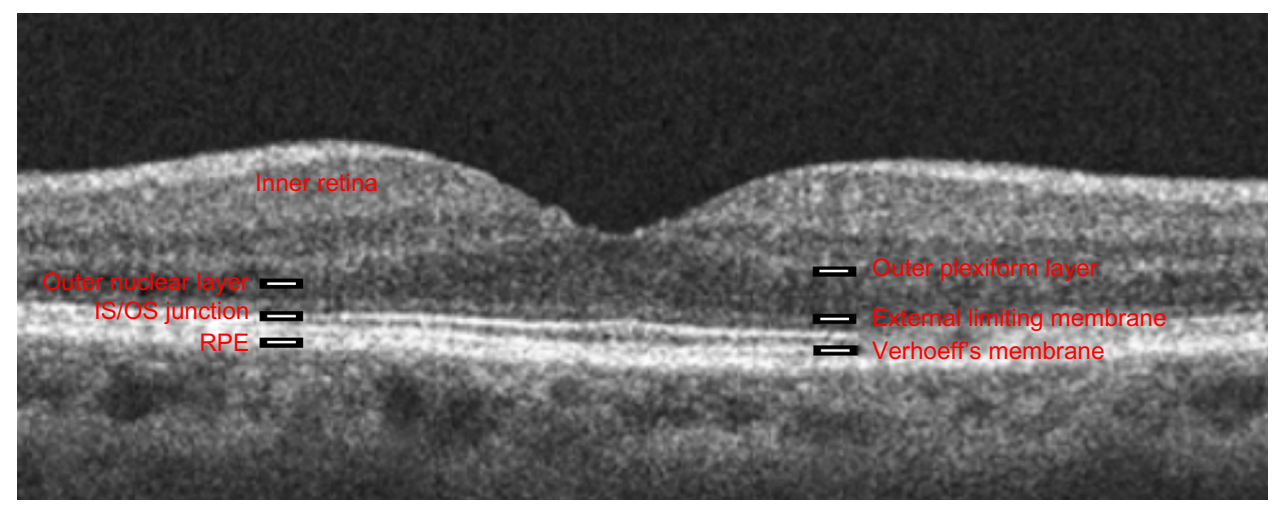

Figure I Spectral-domain optical coherence tomographic image of a human fovea.

Abbreviations: IS/OS, inner segment/outer segment junction of photoreceptors; RPE, retinal pigment epithelium. 
in $7 \%-17 \%$ of participants along with a paradoxical worsening of vision with decreased thickening in $18 \%-26 \%$. The authors concluded that central retinal thickness accounts for only up to $27 \%$ of variability in concurrently measured visual acuity.

Plausible explanations for the seeming lack of direct deterioration of BCVA with increasing OCT-measured macular edema include OCT imaging not being able to account for the duration of edema, changes in the retinal pigment epithelium or other retinal cells that are "OCT-equivalent" or "OCT-lucent", and an inability to categorize macular perfusion/ischemia.

\section{Spectral-domain OCT in management of macular edema}

Some studies have attempted to correlate OCT abnormalities seen in cystoid macular edema with changes seen on fluorescein angiography. ${ }^{5,714-17}$ Despite variance in some study findings, subtle morphologic changes are better appreciated with spectral-domain OCT for retinal or choroidal vascular leakage/hemorrhage and vitreoretinal traction. Therefore, such conditions are often followed by spectral-domain OCT with periodic corroboration by fluorescein angiography or indocyanine green. Arend et al showed that the foveal avascular zone and perifoveal intercapillary area were enlarged in diabetic macular ischemia with reduced BCVA. The visual acuity significantly correlated with the foveal avascular zone $\left(R^{2}=0.51\right)$ and perifoveal intercapillary area $\left(R^{2}=0.24\right)$ without association with cystoid macular edema. ${ }^{18}$ When showing benefit from bevacizumab in central retinal vein occlusion, Moschos and Moschos relied on multifocal electroretinography to show electrophysiologic benefit in OCT-determined "flat" maculas. ${ }^{19}$

Clearly, a "dry" macula is not always normal, and macular edema does not necessitate "poor vision". Additionally, spectral-domain OCT certainly helps in the diagnosis of cystoid macular edema, but can often miss essential findings that aid in the diagnosis. For example, angiography is essential to clarify the cause of cystoid macular edema. While cystoid macular edema (CME) caused by mechanical traction is often best diagnosed with spectral-domain OCT, CME from vascular occlusions (and other causes of macular ischemia), retinal inflammatory conditions, retinal degenerations, and neoplasia obviously require angiography to confirm the diagnosis. In addition, exudative changes can be revealed by leakage on angiography that may not result in formation of cystic spaces best detected by spectral-domain OCT. Similarly, the newest generation of prospective, double-blind, multicenter trials examining the benefit of intravitreal pharmacology to control macular edema have often used macular thickness on OCT only as a retreatment criterion or as a secondary endpoint.

Beginning in 2002 and 2003, triamcinolone acetonide was evaluated by DRCR.net for the treatment of diabetic macular edema. The primary endpoint of BCVA in "pseudophakics" was better with combined treatment, determined in part by OCT criteria. ${ }^{20-22}$ When the effect of cataract formation was eliminated by subanalysis in pseudophakics, maculas with less intraretinal fluid (as determined by OCT) were found to have better vision. Similarly, the RESTORE study (ranibizumab monotherapy or combined with laser versus laser monotherapy for diabetic macular edema) and the DRCR. net study have shown that ranibizumab monotherapy or ranibizumab-deferred laser treatment was superior to triamcinolone monotherapy or standard laser alone with respect to BCVA outcomes over 3 years, likely due to earlier dry maculas (followed by serial OCT images) as a result of treatment with anti-vascular endothelial growth factor. ${ }^{23,24}$

OCT was similarly used as a benchmark for therapeutic efficacy in retinal vein occlusion studies of macular edema using triamcinolone acetonide, eg, SCORE (Standard Care versus COrticosteroid for REtinal Vein Occlusion), ${ }^{20}$ antivascular endothelial growth factor, eg, BRAVO (A Study of the Efficacy and Safety of Ranibizumab Injection in Patients With Macular Edema Secondary to Branch Retinal Vein Occlusion), CRUISE (A study of efficacy and safety of Ranibizumab [Lucentis] injections in patients with macular edema secondary to CRVO), COPERNICUS (Controlled Phase III Evaluation of Repeated Intravitreal administration of VEGF Trap-Eye In Central retinal vein occlusion: Utility and Safety), and GALILEO (General Assessment Limiting Infiltration of Exudates in central retinal vein Occlusion with VEGF Trap-Eye), and ozurdex, eg, Global Evaluation of implaNtable dExamethasone in retinal Vein occlusion with macular edemA (GENEVA). These studies and many others, eg, TDMO (Intravitreal Triamcinolone for Clinically Significant Diabetic Macular Oedema That Persists After Laser Treatment), PACORES (Pan-American Collaborative Retina Study Group), and RESOLVE (Safety and Efficacy of Ranibizumab in Diabetic Macular Edema With Center Involvement), have shown benefit in terms of BCVA from a “dry" macula determined by an OCT.

\section{Spectral-domain OCT prognosis in macular edema}

We are only beginning to understand how chronic cystoid macular edema can lead to a poor prognosis and permanent 
visual loss. Our recent study has addressed how some patients with Irvine-Gass syndrome (pseudophakic cystoid macular edema) recover to 20/20 vision while others suffer permanent vision loss (publication under review). Both groups appear to be the same by funduscopic examination and fluorescein angiography. We used high-resolution spectral-domain OCT to assess for residual structural changes of the retina in both groups of patients. Interestingly, in those with vision loss, we found damage to the cone outer segment tips, which are part of Verhoeff's membrane.

The only prospective, double-blind trial that has evaluated the difference in clinical outcome between time-domain and spectral-domain OCT, that the authors are aware of, is the second-year results of the CATT (Comparison of AMD Treatments Trials) study. ${ }^{25,26}$ BCVA outcomes were followed by treatment differences (pro re nata [PRN] intravitreal injection of anti-vascular endothelial growth factor) for determination of recurrence of macular fluid between time-domain OCT and spectral-domain OCT. It was hoped that with the change to greater OCT resolution and macular volume imaged in the second year of the study, spectral-domain OCT would improve the primary outcome of BCVA for the PRN bevacizumab and ranibizumab arms compared with the monthly protocols. Surprisingly, the outcomes remained unchanged in the second year.

As clinicians, it makes innate sense that clearer macular images with greater macular volume imaged would herald an earlier diagnosis of macular edema. Many studies have already shown that early diagnosis and treatment of cystoid macular edema corresponds to better visual outcomes. Further, as technology advances and the costs of spectral-domain OCT continue to decrease, the question becomes increasingly controversial. Practices will likely opt for the newer technology, and time-domain OCT is already viewed as obsolete by many clinicians. The crossroads of image resolution and treatment "burden" is very similar to the conundrum of full-body computed tomography in radiology. As ophthalmologists we can now visualize macular edema that is clinically inapparent. Are we subjecting patients and society to costly monthly treatments that, if not administered, would have uncertain long-term visual outcomes? Future studies are needed to clarify these and many other related issues. For example, a prospective study investigating PRN treatment for timedomain versus spectral-domain OCT-determined macular edema (eg, in diabetic retinopathy) could elucidate the effects of increasing OCT resolution on clinical outcome (BCVA, number of injections required, foveal thickness).

\section{Spectral-domain OCT for macular edema in the future}

Ongoing OCT research efforts continue to focus on improving image resolution and speed of acquisition. An ultra high-speed spectral-domain OCT has been reported that acquires images at up to 70,000-312,500 axial scans per second. ${ }^{27}$ The use of image registration to identify changes more accurately in local areas of retinal thickening over time will be assessed, as will the combination of spectral-domain OCT images with other imaging modalities, such as fluorescein angiography, indocyanine green angiography, and confocal scanning laser ophthalmoscopy. Among the new generations of OCT, Spectralis OCT is equipped with image registration as well as an eye-tracking system that is able to monitor the eye position continuously using a light beam, thus increasing the reproducibility of retinal thickness measurements. ${ }^{28}$

Clearly, the future of OCT imaging is bright. The detail with which this technology allows the ophthalmologist to become familiar with anatomic alterations of the pathologic retina (or the developing or aging retina) is nothing short of marvelous.

\section{Disclosure}

The authors report no conflicts of interest in this work.

\section{References}

1. Huang D, Swanson EA, Lin CP, et al. Optical coherence tomography. Science. 1991;254:1178-1181.

2. Srinivasan VJ, Wojtkowski M, Witkin AJ, et al. High-definition and 3-dimensional imaging of macular pathologies with high-speed ultrahigh-resolution optical coherence tomography. Ophthalmology. 2006;113:2054. e2051-e2014.

3. Wolf S, Wolf-Schnurrbusch U. Spectral-domain optical coherence tomography use in macular diseases: a review. Ophthalmologica. 2010;224:333-340.

4. Brown JC, Solomon SD, Bressler SB, Schachat AP, DiBernardo C, Bressler NM. Detection of diabetic foveal edema: contact lens biomicroscopy compared with optical coherence tomography. Arch Ophthalmol. 2004;122:330-335.

5. Soliman W, Sander B, Hasler PW, Larsen M. Correlation between intraretinal changes in diabetic macular oedema seen in fluorescein angiography and optical coherence tomography. Acta Ophthalmol. 2008;86:34-39.

6. Nakajima M, Sakonjyu N, Mori R, Ishihara N, Yuzawa M. Correlation between angiographic and pathological findings in experimental choroidal neovascular membranes after transpupillary thermotherapy. Jpn J Ophthalmol. 2004;48:535-541.

7. Neubauer AS, Chryssafis C, Priglinger SG, et al. Topography of diabetic macular oedema compared with fluorescein angiography. Acta Ophthalmol Scand. 2007;85:32-39.

8. Schaudig U, Scholz F, Lerche RC, Richard G. Optical coherence tomography for macular edema. Classification, quantitative assessment, and rational usage in the clinical practice. Ophthalmologe. 2004;101: 785-793. German.

9. Ota M, Tsujikawa A, Murakami T, et al. Association between integrity of foveal photoreceptor layer and visual acuity in branch retinal vein occlusion. Br J Ophthalmol. 2007;91:1644-1649. 
10. Smith AJ, Telander DG, Zawadzki RJ, et al. High-resolution Fourierdomain optical coherence tomography and microperimetric findings after macula-off retinal detachment repair. Ophthalmology. 2008;115: 1923-1929.

11. Telander DG, Choi SS, Zawadzki RJ, Berger N, Keltner JL, Werner JS. Microstructural abnormalities revealed by high resolution imaging systems in central macular arteriovenous malformation. Ophthalmic Surg Lasers Imaging. 2010:1-4.

12. Otani T, Kishi S, Maruyama Y. Patterns of diabetic macular edema with optical coherence tomography. Am J Ophthalmol. 1999;127: 688-693.

13. Baskin DE. Optical coherence tomography in diabetic macular edema. Curr Opin Ophthalmol. 2010;21:172-177.

14. Ozdek SC, Erdinc MA, Gurelik G, Aydin B, Bahceci U, Hasanreisoglu B. Optical coherence tomographic assessment of diabetic macular edema: comparison with fluorescein angiographic and clinical findings. Ophthalmologica. 2005;219:86-92.

15. Kang SW, Park CY, Ham DI. The correlation between fluorescein angiographic and optical coherence tomographic features in clinically significant diabetic macular edema. Am J Ophthalmol. 2004;137: 313-322.

16. Ghazi NG, Ciralsky JB, Shah SM, Campochiaro PA, Haller JA. Optical coherence tomography findings in persistent diabetic macular edema: the vitreomacular interface. Am J Ophthalmol. 2007;144:747-754.

17. Tran TH, de Smet MD, Bodaghi B, Fardeau C, Cassoux N, Lehoang P. Uveitic macular oedema: correlation between optical coherence tomography patterns with visual acuity and fluorescein angiography. Br J Ophthalmol. 2008;92:922-927.

18. Arend O, Wolf S, Harris A, Reim M. The relationship of macular microcirculation to visual acuity in diabetic patients. Arch Ophthalmol. 1995;113:610-614.

19. Moschos MM, Moschos M. Intraocular bevacizumab for macular edema due to CRVO. A multifocal-ERG and OCT study. Doc Ophthalmol. 2008;116:147-152.
20. Martidis A, Duker JS, Greenberg PB, et al. Intravitreal triamcinolone for refractory diabetic macular edema. Ophthalmology. 2002;109: 920-927.

21. Jonas JB, Kreissig I, Sofker A, Degenring RF. Intravitreal injection of triamcinolone for diffuse diabetic macular edema. Arch Ophthalmol. 2003;121:57-61

22. Beck RW, Edwards AR, Aiello LP, et al. Three-year follow-up of a randomized trial comparing focal/grid photocoagulation and intravitreal triamcinolone for diabetic macular edema. Arch Ophthalmol. 2009;127: 245-251.

23. Elman MJ, Bressler NM, Qin H, et al. Expanded 2-year follow-up of ranibizumab plus prompt or deferred laser or triamcinolone plus prompt laser for diabetic macular edema. Ophthalmology. 2011;118: 609-614.

24. Mitchell P, Bandello F, Schmidt-Erfurth U, et al. The RESTORE study: ranibizumab monotherapy or combined with laser versus laser monotherapy for diabetic macular edema. Ophthalmology. 2011;118. 615-625.

25. Decroos FC, Toth CA, Stinnett SS, Heydary CS, Burns R, Jaffe GJ. Optical coherence tomography grading reproducibility during the comparison of age-related macular degeneration treatments trials. Ophthalmology. 2012;119:2549-2557.

26. Martin DF, Maguire MG, Fine SL, et al. Ranibizumab and bevacizumab for treatment of neovascular age-related macular degeneration: two-year results. Ophthalmology. 2012;119:1388-1398.

27. Potsaid B, Gorczynska I, Srinivasan VJ, et al. Ultrahigh speed spectral/ Fourier domain OCT ophthalmic imaging at 70,000 to 312,500 axial scans per second. Opt Express. 2008;16:15149-15169.

28. Chin EK, Sedeek RW, Li Y, et al. Reproducibility of macular thickness measurement among five OCT instruments: effects of image resolution, image registration, and eye tracking. Ophthalmic Surg Lasers Imaging 2012;43:97-108
Clinical Ophthalmology

\section{Publish your work in this journal}

Clinical Ophthalmology is an international, peer-reviewed journal covering all subspecialties within ophthalmology. Key topics include: Optometry; Visual science; Pharmacology and drug therapy in eye diseases; Basic Sciences; Primary and Secondary eye care; Patien Safety and Quality of Care Improvements. This journal is indexed on

\section{Dovepress}

PubMed Central and CAS, and is the official journal of The Society of Clinical Ophthalmology (SCO). The manuscript management system is completely online and includes a very quick and fair peer-review system, which is all easy to use. Visit http://www.dovepress.com/ testimonials.php to read real quotes from published authors. 